\title{
DOOCUMENOS
}

\section{0 professor Miguel Reale na Conferência Internacional do Trabalho de 1951}

Em 1951, o Professor Miguel Reale foi nomeado, pelo Presidente Getúlio Vargas, para, na qualidade de Delegado Governamental, representar o Brasil na $34 .^{a}$ sessão da Organização Internacional do Trabalho (oIT) realizada em Genebra, em junho daquele ano.

$O$ assunto fundamental em pauta era o problema do salário mínimo do trabalhador agrícola.

No seio da Comissão Especial, o Professor Reale apresentou emenda supressiva do art. $6 .^{\circ}$ do Projeto, o qual pretendia abolir o salário mínimo nas áreas de grandes plantações, tal como era pretendido pelas potências colonialistas que exploravam a Ásia e a África.

Além das razões de justiça social, a proposição representava grande ameaça para o Brasil, no plano do comércio internacional, visto como seríamos obrigados a competir com plantadores de café, de açúcar e de algodão, naqueles dois continentes, usufruindo êstes de mão de obra a preço vil.

Vencido na Comissão, o Professor Reale transferiu os debates para o plenário da conferência, dando seu apoio à Convenção, mas com ressalva do malsinado art. $6^{\circ}$.

O discurso do Professor Reale, proferido na sessão de 25 de junho, produziu grande sensação, sendo acompanhado em sua repulsa pelos Delegados Governamentais, patronais e dos trabalhadores da América Latina (êstes apoios constam dos discursos dos Delegados do México e da Argentina, Marquez de Alba e Rosalez) bem como dos Paises da Cortina de Ferro (Licki da Polônia, Vavricka 
da Chécoslováquia), e também dos Países Árabes. Com fyrande surpresa os colonialistas foram derrotados.

Em verdade, em razão do discurso do Delegado Brasileiro, adiante transcrito, foi rejeitado o art. $6 .^{\circ}$ por 68 votos contra 59, o que demonstra a atenção suscitada pelo problema.

A vitória do eminente mestre brasileiro alcançou grande repercussão, sendo aprovado requerimento, na Assembléia Legislativa do Estado, subscrito, entre outros, pelos Deputados Jânio da Silva Quadros, Pinheiro Júnior, Castro Neves, Araripe Serpa, para que fôsse aclamada a seguinte moção:

"O Brasil acaba de conquistar, na Conferência Internacional do Trabalho, presentemente reunida em Genebra, uma vitória brilhante: por 68 votos contra 59, após longos debates, foi aprovada a tese contrária à isenção, pleiteada especialmente pela India e Paquistão, no sentido de não ser concedido salário mínimo a seus trabalhadores agrícolas.

O delegado brasileiro Prof. Miguel Reale, sustentou que duas razões se opunham a essa pretensão: $10^{\circ}$ ) privar um trabalhador do salário mínimo é violar a Declaração - de Direito Social de Filadélfia; $2^{\circ}$ ) é estabelecer desigualdade no campo internacional, onerando a mão de obra de uns em contraposição a outros Países, que não satisfazem os encargos sociais.

Opôs-se o representante do Brasil ao "colonialismo de 2. grau" que se desejava estabelecer, declarando que já era exorbitante a faculdade que têm as nações de não aplicar as convenções nos territórios coloniais. O plenário, aceitando o ponto de vista defendido pelo Prof. Reale, rejeitou o art. $6^{\circ}$, que visava dar a determinados Países a faculdade de não aplicar a Convenção sôbre o salário minimo do trabalhador agrário, no próprio território.

Sem dúvida alguma o trabalho desenvolvido pela delegação brasileira à Conferência Internacional do Trabalho yem provar que a escôlha dos representantes do Brasil feita pelo sr. Presidente da República foi das mais felizes 
e profícuas, motivo pelo qual submeto à aprovação de meus nobres pares etc."

("Diário Oficial do Estado", de 7 de julho de 1951, pg. 30).

$\mathrm{Na}$ oportunidade, falaram os Deputados Araripe Serpa e Porfírio da Paz, tendo aquêle dito estas palavras finais:

“Assim, o Professor Miguel Reale conquistou para o Brasil grande vitória, um grande feito, provando ao mundo a capacidade, o brilho da cultura brasileira"

É de notar-se que as rejeições de artigos no seio do Plenário da 0.I.T. são rarìssimas, excepcionais mesmo.

Eis o texto do discurso que suscitou a exclusão do art. $6^{\circ}$ da Convenção sôbre salário do trabalhador rural;

- "M. Reale (délégué gouvernemental, Brésil) - Nous devons dire, tout d'abord, que la délégation du Brésil approuve le projet de convention concernant les méthodes de fixation des salaires minima dans l'agriculture, mais elle se permet de faire des remarques qui ont pour but de préserver certains principes fondamentaux solennellement proclamés à Philadelphie par la Confẻrence générale de l'Organisation Internacionale du Travail. La déclaration qui fut alors adoptée reconnaissait le droit à un salaire minimum vital pour tous ceux qui ont un employ et ont besoin d'une telle protection. Ce principe ne peut, évidemment, justifier une disposition telle que l'article $6 \mathrm{du}$ projet qui nous préoccupe, dont le résultat pratique serait I'exclusion de millions de travailleurs du bénéfice d'un salaire minimum.

"Il faut noter tout d'abord que l'article $6 \mathrm{du}$ projet de convention dont nous sommes saisis ne concerne pas les régions des territoires non métropolitains ou sous tutelles pour lesquels sont prévues des dispositions exceptionnelles par l'article 35 de la Constitution de l'o.I.T. $\mathrm{Ce}$ que prétend le texte du projet, c'est exempter de l'applicafion de la convention certaines régions des territoires métropolitains eux-mêmes, et cela nous semble incompatible 
avec le programme d'action de notre Organisation, surtout lorsque nous nous rendons compte du caractère général d'une autre convention qui a déjá assuré le droit au salaire minimum à tous les travailleurs.

"Qu'il nous soit permis de rappeler que le texte actuel de l'article 6 du projet a été proposé par le Bureau International du Travail afin de répondre à des difficultés soulevées, après la première discussion qui a eu lieu, l'an dernier, au sein de cette Conférence. Cependant, nous júgeons que la solution proposée constituerait une modification fondamentale aux conclusions déjà adoptées.

"Nous n'ignorons pas qu'il existe une convention concernant les salaires minima dans toutes les branches de la production, et c'est précisément pour ce motif que l'article $6 \mathrm{du}$ projet, dans sa rédaction actuelle, ne pourrait être satisfaisant. En effet, cet article, beaucoup trop général, permettrait des interprétations abusives qu'il importe de prévenir.

“Tout en reconnaissant la nécessité de donner à la convention toute la souplesse nécessaire pour faciliter l'action législative en cette matière, il convient de déclarer, de façon claire et précise, que, dans l'impossibilité d'appliquer des méthodes de fixation des taux des salaires minima prévues par le texte de la convention, les gouvernements seraient tenus d'avoir recours à d'autres procédés afin d'assurer, à tous les travailleurs, le salaire minimum vital.

"Nous devons conserver toujours à l'esprit le droit intangible des travailleurs au juste prix de leur participation dans la création des biens économiques. C'est pour ce motif que la délégation brésilienne émettra des réserves, bien que votant en faveur du projet de convention.

"Selon l'article 35 de la Constitution de l'o.r.T., tous les Etats Membres "s'engagent à appliquer les conventions qu'ils auront ratifiées. aux territoires non métropolitains dont ils assument les relations internationales, y compris tous territoires sous tutelle pour lesquels ils seraient l'au- 
torité chargée de l'administration, à moins que les questions traitées par la convention ne rentrent dans le cadre de la compétence propre des autorités du territoire ou que la convention ne soit rendue inapplicable par les conditions locales, ou sous réserve des modifications qui seraient nécessaires pour adapter les conventions aux conditions locales". La règle générale est donc l'extension des conventions ratifiées aux territoires non métropolitains euxmêmes, pour lesquels sont néanmoins admises des exemptions.

"Il faut, d'autre part, interpréter le dit article 35 de la Constitution de l'o.I.T. à la lumière du paragraphe $V$ de la Déclaration de Philadelphie, qui, tout en tenant compte du degré de développement social et économique de chaque peuple, reconnaît que l'application progressive des conventions aux peuples libres ou qui viennent d'acquérir leur souveraineté, intéresse l'ensemble du monde civilisé.

"Nous nous demandons si le texte actuel de l'article 6 du projet répond, non seulement à l'esprit, mais à la lettre même de la Déclaration de Philadelphie.

"Monsieur le Président, si des exemptions peuvent être autorisées pour les territoires dépendants, nous ne pouvons les concevoir ou les admettre sans formuler de réserve en ce qui concerne les territoires des peuples indépendants. L'extension des règles exceptionnelles de l'article 35 de la Constitution de l'o.r.t. aux territoires métropolitains constituerait une anomalie, et son résultat positif serait le maintien de certains systèmes de rémunération du travail dangereux pour tous. Nous ne devons pas oublier que, selon les termes précis rappelés dans la Déclaration de Philadelphie, "une paix durable ne peut être établie que sur la base de la justice sociale". Dans ces conditions, on peut affirmer que les charges sociales, notamment celles qui se rapportent au minimum nécessaire à la subsistance humaine, constituent la contre partie naturelle de la souveraineté. 
"Si le salaire minimum n'est que la limite au dessous de laquelle il n'est pas possible de songer à une forme quelconque de travail libre, en harmonie avec la dignité de l'homme, nous nous rendons tous compte de la portée de l'exemption prévue par le texte actuel de l'article 6 . Nous devons éviter qu'on ne puisse attribuer, dans un avenir plus ou moins rapproché, à cette Conférence, la sesponsabilité d'avoir privé pratiquement des millions de travailleurs du bénéfice du salaire minimum.

"On pourra nous objecter que la situation spéciale de certaines régions du monde oppose des difficultés d'ordre technique à l'application de toutes les dispositions de la convention sur laquelle nous devons nous prononcer aujourd'hui. Nous sommes convaincus de la complexité de ce problème, mais si les règles d'une convention ne peuvent parfois être appliquées dans leur ensemble, il faudra tout de même préserver certains droits essentiels au travail libre. Nous n'avons pas besoin de nous attarder sur cette question pour démontrer à cette Auguste Conférence que la garantie effective du salaire minimum est la condition primordiale de la justice sociale.

“D'autre part, le projet de convention, dans ses autres articles, prévoit déjà, avec beaucoup de souplesse et de prudence, les difficultés qu'on pourrait opposer à notre point de vue. Il nous suffit de lire le texte du paragraphe 2 de l'article premier pour vérifier qu'est assurée à chaque Membre la faculté de déterminer les entreprises, les occupations et les catégories de personnes auxquelles seront appliquées les méthodes de fixation des salaires minima. Aux termes du paragraphe 3 du même article, l'autorité compétente pourra aussi exclure certaines catégories de personnes de l'application des règles prévues dans la convention.

"Avec ces dispositions, a-t-on réellement besoin d'établir l'importante et grave exemption prévue par l'article 6? Nous ne .le croyons pas. Personne, en l'état actuel du progrès des sciences sociales, ne pourra affirmer qu'il 
est tout à fait impossible de trouver des méthodes de fixation des taux des salaires minima plus ou moins appropriées à telle ou telle autre région du monde. L'o.I.T. ne se refusera pas, sans doute, à aider les gouvernements qui auront besoin de la contribution toujours précieuse de ses experts. En ce qui concerne le Brésil, nous pouvons dire que le système de discrimination des zones de salaire nous assure l'adaptation du taux de la rémunération du travail au coût de la vie dans les différentes régions et localités, moyennant l'action complémentaire du gouvernement fédéral et des commissions régionales, instituées sur des bases paritaires par les représentants des employeurs et des travailleurs.

"Avant de terminer, je me permettrai de faire encore quelques remarques. Une convention, une fois ratifiée, s'insère dans le système de la législation de chaque Etat Membre, de sorte que ses dispositions ont force dérogatoire à l'égard de tous les préceptes qui lui sont contraires. Il faut, par conséquent, que la convention ne représente pas une régression par rapport à des principes déjà reconnus par chaque système national de droit. Dans le cas spécial du Brésil, je dois dire que la garantie du salaire minimum pour tous les habitants de notre pays, quel que soit leur sexe, leur occupation ou leur nationalité, est désormais une garantie constitutionnelle. L'article $157 \mathrm{de}$ la Constitution brésilienne de 1946 n'a d'ailleurs fait que sanctionner notre tradition à cet égard. L'exemption prévue par le teste actuel de l'article 6 représenterait, par conséquent, une solution s'opposant au progrès que nous avons déjà atteint dans les domaines du droit social.

“D'autre part, il faut encore observer que deux autres problèmes doivent être mis nettement en évidence l'un par rapport à l'autre. Si, d'une part, nous réaffirmons notre volonté de consolider et de perfectionner nos lois visant à protéger les travailleurs ruraux, d'autre part, nous espérons que des principes legislatifs semblables seront consacrés et appliqués sur toute la surface de la terre, afin 
d'éliminer toute concurrence déloyale due au renchérissement unilatéral de notre main-d'oeuvre.

J'espère avoir donné les raisons pour lesquelles la délégation brésilienne votera le projet de convention, sous réserve de l'article 6 , tout en déclarant que nous n'avons aucune intention d'utiliser la clause dérogatoire qui y est prévue, fidèles en cela à l'esprit de justice sociale dont s'inspire l'Organisation Internationale du Travail.

(Conférence Internationale du Travail Compte rendu provisoire - Trente-quatrième session, Genève Dix-huitième Séance Lundi, 25 juin 1951) 